\title{
Cort-X II: low power element design of a large-scale spatio-temporal pattern clustering system
}

\author{
Jie Yuan*, Ning Song, Nabil Farhat, Jan Van der Spiegel \\ ESE Department \\ University of Pennsylvania \\ Philadelphia, PA, USA \\ eeyuan@ece.ust.hk; \{ningsong, farhat, jan\}@seas.upenn.edu
}

\begin{abstract}
Complex spatio-temporal patterns can be clustered using a network of parametrically coupled logistic maps. This paper describes the processing element design of such a Cort-X system. Each Cort-X element consists of a non-linear coupling (LC) and a non-linear dynamic element (IRON). The circuits are designed for low-power operation and to be robust against process variations. This has been accomplished by using openloop circuits, and a self-calibration technique that compensate for process variations. The circuits were implemented in a 0.25 um, 2.5V CMOS process and consumes a total of $12 \mathrm{~mW}$ of power at $1 \mathrm{MHz}$ which is about a factor of 20 less power than previous realizations. This opens the possibility for building a large-scale Cort-X system on a chip for the recognition of complex spatio-temporal patterns.
\end{abstract}

\section{INTRODUCTION}

Neurophysiologic study suggests that the biological cortex is organized hierarchically at the macroscopic level and modularly at the microscopic level [1]. At the microscopic level, a cortical patch is composed of a modular network of cortical columns. It has been shown that a network of parametrically coupled logistic maps (PCLMN) can model the biological structure of the cortical patch [2]. Through system-level simulations, the PCLMN shows superior capability for clustering spatio-temporal patterns (STP). The PCLMN also has advantages of network capacity and hardware implementation, which paves the way to a large-scale intelligent system for STP recognition. The system can be useful for applications such as epileptic seizure anticipation, and automated target recognition (ATR).

A small PCLMN with five processing elements (PEs) is given in Fig. 1. The PEs are coupled through nonlinear interconnections. Each $\mathrm{PE}$ in the network includes an interconnection module and a 1-D parametrically coupled/driven logistic map. The 1-D logistic map can be generated by an integrated-circuit relaxation oscillator neuron (IRON) [3].

The PCLMN has been previously designed in CMOS circuits in a $0.6 \mathrm{~m}$ 5V CMOS process [5] and [6], which is

* Jie Yuan is current at ECE department of Hong Kong Unive. of Sci. \& Tech

This research was supported by the Office of Naval Research under grant No. N00014-94-1-0931, and by Army Research Office grants: MURI DAAD-19-01-1-0603 via Georgia Institute of Technology, and DURIP W911NF-04-1-0177.

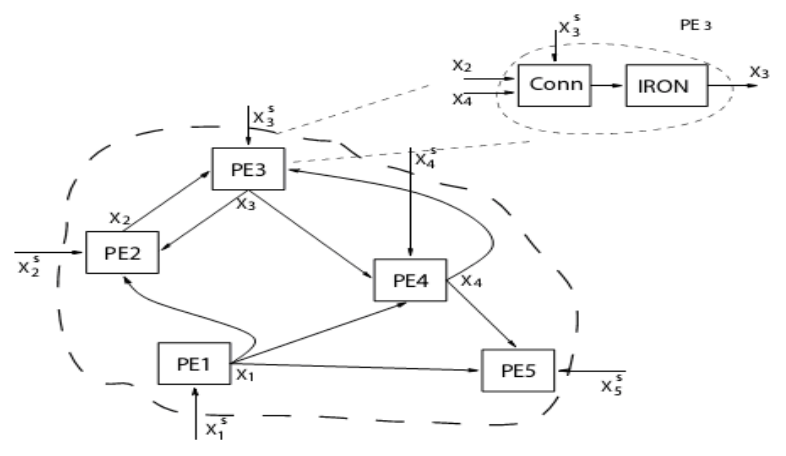

Figure 1. A 5-element parametrically coupled logistic map network

named Cort-X I [4]. Although Cort-X I was able to achieve good system-level results, the power consumption is high. This was in part due to the use of feedback to implement the nonlinear functions.

For large-scale integration of a Cort-X system, a lowpower design is needed. Open-loop circuits and new structures are used to reduce power consumption. To compensate for the process variations for which the openloop circuits are more sensitive, a self-calibration technique is developed. Cort-X II was fabricated in a $0.25 \mathrm{um} 2.5 \mathrm{~V}$ CMOS process. In this paper, the CMOS design and measurements of the new Cort-X II element is presented.

\section{THE CORT-X ELEMENT}

Inside a PE, as shown in Fig. 2, the Local Coupling (LC) module implements the nonlinear interconnection and controls the dynamics of the nonlinear element IRON, by generating the bifurcation parameter $u$.

\section{A. IRON}

As presented in [5], the IRON model is similar to an integrate-and-fire neuron. As illustrated in Fig. 3, the dynamics of an IRON is limited by a threshold voltage $\left(V_{t}\right)$ and a rest potential $\left(\mathrm{V}_{\mathrm{r}}\right)$. The membrane potential $\left(\mathrm{V}_{\mathrm{m}}\right)$ builds up at a constant rate. When $\mathrm{V}_{\mathrm{m}}$ reaches $\mathrm{V}_{\mathrm{t}}$, the IRON fires, 


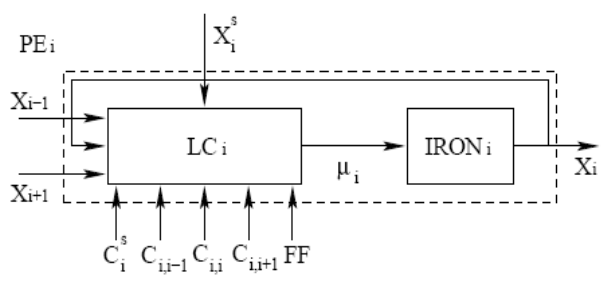

Figure 2. The structure of element $\mathrm{PE}_{\mathrm{i}}$, with local interconnection for $\mathrm{i}$-th IRON.

and $\mathrm{V}_{\mathrm{m}}$ descends to $\mathrm{V}_{\mathrm{r}}$ within a refractory period $\left(\mathrm{T}_{\mathrm{r}}\right)$. With a periodic rest potential, the phase of the pulse sequence $\left\{\mathrm{t}_{\mathrm{i}}\right\}$ fired by the IRON conforms to a 1-D map. It can be verified [5] that the IRON can generate the 1-D logistic map with the driving waveform in Eqn. 1.

$$
V_{r}(t)=V_{a} \frac{t}{T}-4 \mu \frac{t}{T}\left(1-\frac{t}{T}\right) V_{a}+V_{b}, \mu \in[0,1]
$$

\section{B. Parametrical Coupling}

As presented in [6], the parametrical coupling of the i-th $\mathrm{PE}$ is nonlinearly modulated by both external and internal influences according to the expression,

$$
\mu_{i}(t)=e^{-\alpha t} g\left(X_{i}^{s}, C_{i}^{s}\right)+\frac{1-e^{-\alpha t}}{N_{i}} \sum_{j \in\left\{N_{i}\right\}} g\left(X_{j}, C_{i j}\right),
$$

where $\mathrm{X}_{\mathrm{i}}^{\mathrm{s}}$ is the external stimulus for the $\mathrm{i}$-th IRON, $\mathrm{X}_{\mathrm{j}}$ is the output from the $\mathrm{j}$-th IRON, $\mathrm{C}_{\mathrm{i}}^{\mathrm{s}}$ is the coupling parameter for the external stimulus of the $\mathrm{i}$-th IRON, and $\mathrm{C}_{\mathrm{ij}}$ is the coupling parameter from the $\mathrm{j}$-th IRON to the $\mathrm{i}$-th IRON.

The first term in Eqn. 2 represents the influence of the external input, while the second term is the average effect from IRONs inside the network. $\mathrm{e}^{-\alpha t}$ represents a forgetting factor (FF), while 1-e $\mathrm{e}^{-\alpha t}$ is the complement. As the network adapts, FF exponentially decreases. As a result, the external influence gradually gives way to the internal influence. During this transition, the network accepts the external STP and, ultimately, adapts itself to an internal attractor. The nonlinear $\mathrm{g}($ ) function is in Eqn. 3 .

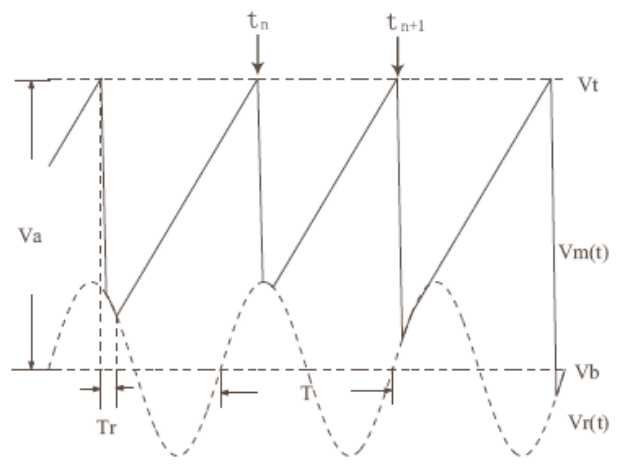

Figure 3. The dynamics of a sine-circle IRON with sinusoidal driving waveform

$$
g(X, C)=X^{C}, \quad X \in[0,1]
$$

\section{CORT-X II CIRCUIT DESCRIPTION}

Cort-X II is primarily designed to reduce the power consumption of Cort-X I, which is described in [5] and [6].

\section{A. IRON Circuit}

The IRON circuit of Cort-X I is a spike-based design [5], shown in Fig. 4. When $V_{m}$ charges beyond $V_{t}$, the circuit generates a narrow pulse, which switches the membrane capacitor $\mathrm{C}_{\mathrm{r}}$ to the rest potential generation circuit, so that $\mathrm{V}_{\mathrm{m}}$ is discharged to $\mathrm{V}_{\mathrm{r}}$. After the pulse, $\mathrm{C}_{\mathrm{r}}$ is switched back to the reference current to start a new charging cycle. Hence, the discharging process of $\mathrm{C}_{\mathrm{r}}$ is limited to the width of the narrow pulse, which is designed to be 20ns. This narrow pulse requires a high speed OPAMP in order to discharge the capacitor fast enough. As a result about half of the power consumption of the IRON is due to the amplifier [5].

A new designed two-phase IRON, shown in Fig. 5, avoids narrow pulses. In this IRON, two branches operate alternatively. The phase detector generates charging phases $\mathrm{S} 1$ and S2 based on the decisions from the two comparators. During S1 phase, branch 1 is charging, and branch 2 is discharging. During S2 phase, the branches switch their roles. The advantage of the two-phase scheme is that the discharging phase for both branches is considerably longer than the 20ns pulse in Cort-X I. Fig. 6 is an illustration of this. The transitions of S1 and S2 are the moments that the IRON is supposed to fire a pulse. For the current two-phase scheme, the length of S1 and S2 phases are indeed the distance between two pulses, which is about lus in normal operation. Therefore, the driving requirement on the rest potential generation circuit is considerably relaxed, which allowed us to eliminate the power-hungry amplifier of the previous version.

Another major factor underlying power consumption in the IRON in Cort-X I is the closed-loop S/H deck [5], which accounts for about $25 \%$ of the power consumed by the IRON. Since the resolution requirement of the IRON is 10bits, in the current design, open-loop sampling is used.

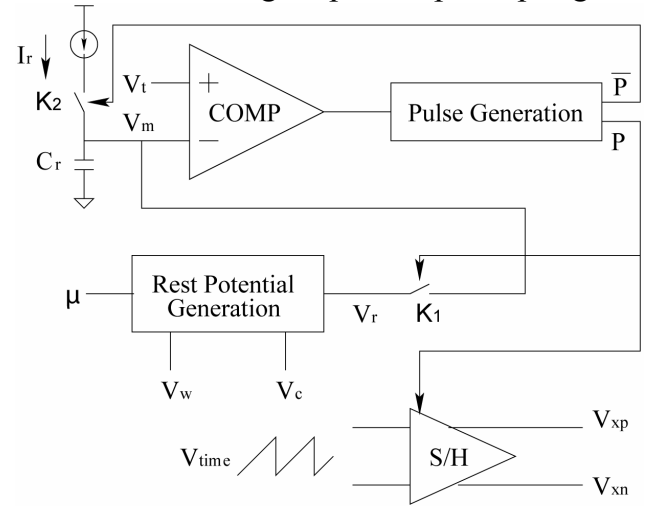

Figure 4. The block diagram of the IRON circuit in Cort-X I 
As a result, the power of the newly designed IRON is reduced to $2 \mathrm{~mW}$ from the original $40 \mathrm{~mW}$ level.

\section{B. LC Circuit}

The local coupling (LC) circuit in Cort-X I [6] consists mainly of feedback circuits, which requires OPAMPs. To reduce the power consumption, the nonlinear $\mathrm{g}()$ function in Eqn. 3 is designed with only feedforward circuits, while the overall function in Eqn. 2 is implemented by current mode circuits.

The newly designed $\mathrm{g}()$ function is shown in Fig. 7. It is composed of a logarithmic circuit, a voltage multiplier and an exponential circuit similar to the one in Cort-X I. Using the BSIM3 model as in [6], the transfer function of the circuit, Eqn. 4, can be obtained, assuming good matching between M3 and M11, which are weakly inverted.

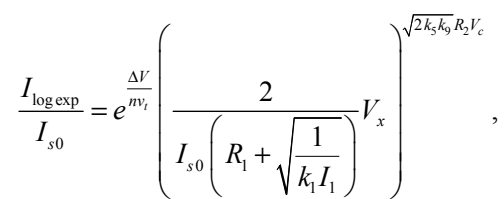

Here $I_{s 0}$ is the leakage current of M3 and M11; $v_{t}$ is the thermal voltage; $\mathrm{n}$ is the swing factor; $\Delta \mathrm{V}$ is the voltage difference between the biasing voltage $\mathrm{V}_{\mathrm{tdr}}$ and the threshold voltage $\mathrm{V}_{\mathrm{T}}$ of $\mathrm{M} 3$ and $\mathrm{M} 11$.

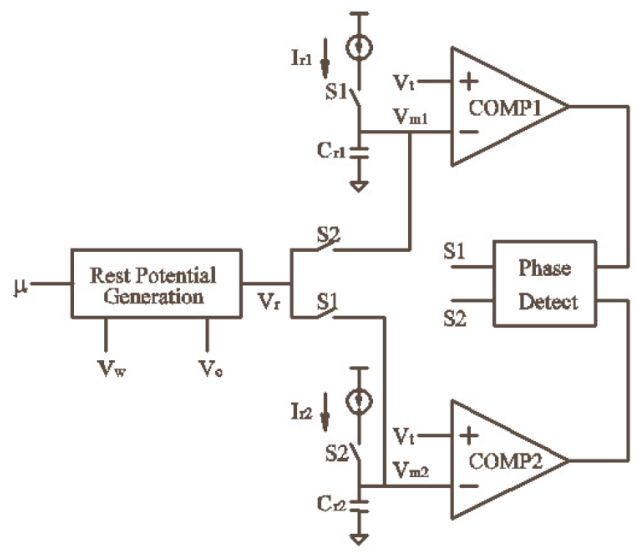

Figure 5. The two-phase IRON in Cort-X II

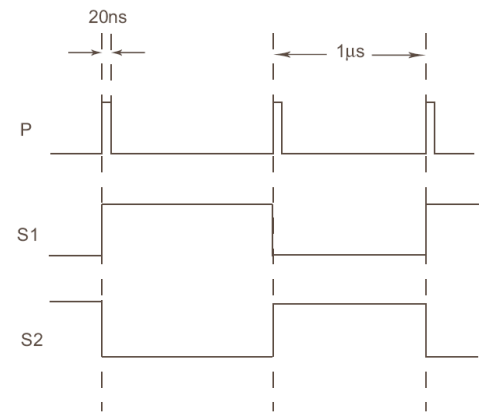

Figure 6. The length of S1 and S2 phases are considerably longer than the $20 \mathrm{~ns}$ pulse width

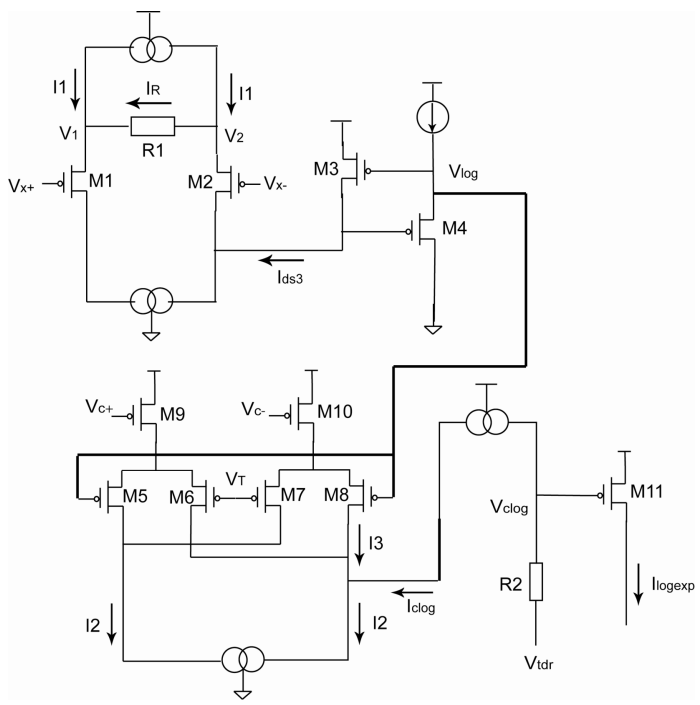

Figure 7. The circuit for $\mathrm{g}()$ function in Cort-X II

The parametrical power function in Eqn. 4 is process dependent. In order to remove this dependency, a selfcalibration technique is implemented in the $\mathrm{g}()$ function circuit in Fig. 7. The calibration procedure operates in two phases. During the calibration phase, the maximum input $\mathrm{V}_{\mathrm{xmax}}$ is applied to the circuit. The output $\mathrm{V}_{\log }$ from the logarithmic module is stored. During the normal operation phase, the stored value of $\mathrm{V}_{\log }$ serves as the $\mathrm{V}_{\mathrm{T}}$ for the conversion. One can derive the transfer function shown in Eqn. 5 from this calibration scheme. Because the process dependent $\mathrm{I}_{\mathrm{s} 0}$ can be tuned out by adjusting $\mathrm{V}_{\mathrm{tdr}}$, the process variation can be effectively removed by this self-calibration procedure.

$$
I_{\log \exp }=\left(I_{s 0} e^{\frac{\Delta V}{n_{t}}}\right)\left(\frac{V_{x}}{V_{x \max }}\right)^{\sqrt{2 k_{s} k_{g}} R_{2} V_{c}}
$$

Since the output of the $\mathrm{g}()$ function circuit is a current, current mode processing becomes a more power-efficient method for the overall LC module, shown in Fig. 8.

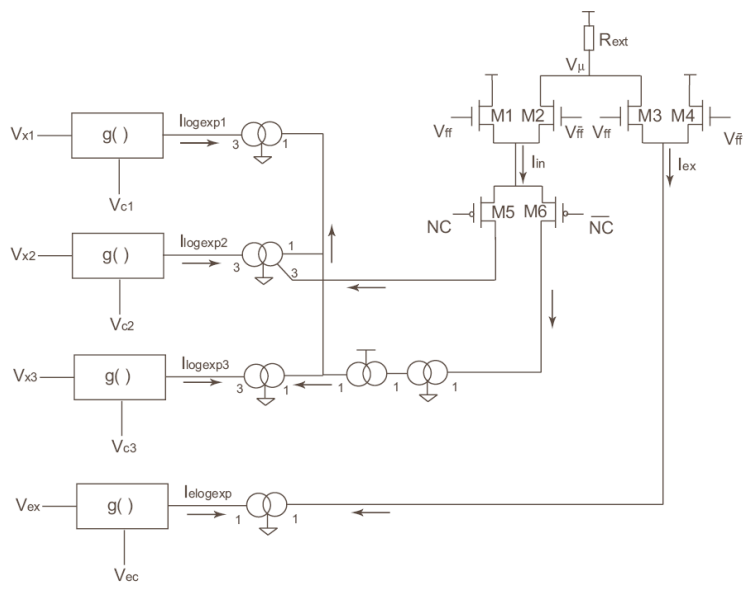

Figure 8. The current mode LC module 


\section{EXPERIMENTAL RESULTS}

The die photo of the Cort-X II chip is shown in Fig. 9. The active die area of the chip is $3 \mathrm{~mm} \times 2.3 \mathrm{~mm}$, which includes $16 \mathrm{LCs}$ and 16 IRONs. Each LC circuit occupies $0.4 \mathrm{~mm} \times 0.46 \mathrm{~mm}$ and consumes $10 \mathrm{~mW}$. Each IRON has a dimension of $0.17 \mathrm{~mm} \times 0.22 \mathrm{~mm}$ and consumes $2 \mathrm{~mW}$.

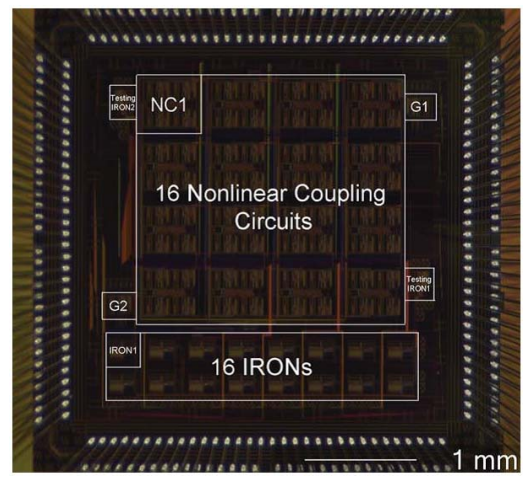

Figure 9. The die photo of the Cort-X II chip

A 6-layer testing board has been designed to characterize the chip. The transfer function of the nonlinear $g()$ function is measured, and plotted in Fig. 10. The measurements show that the nonlinear coupling circuit is able to accurately implement the function $\mathrm{g}()$.

The IRON has been tested for its dynamic performance at $1 \mathrm{MHz}$. Fig. 11 shows the bifurcation diagram measured for the logistic map. Although phase noise is present in the diagram, bifurcations between the different characteristic types of dynamics are evident. We expect that a significant amount of noise is due to the test board. We are now developing a low-noise testing board. This improved setup will reduce the phase noise of the IRON significantly.

\section{CONCLUSIONS}

Spatio-temporal patterns can be classified using parametrically coupled logistic maps. This paper describes a new Cort-X element needed to realize such a system on a chip. It consists of two components: the non-linear local coupling element (LC) and the dynamic element (IRON). By using open-loop circuits together with a self-calibration technique, we were able to compensate for process variations and reduce the power consumption by as much as 20 times as compared to the previous version of Cort-X.

A Cort-X II chip is fabricated in a $0.25 \mathrm{um} 2.5 \mathrm{~V}$ CMOS process. Each element consumes $12 \mathrm{~mW}$ power. Test results show that the nonlinear coupling circuits implement good nonlinear transfer function with fast settling. These elements

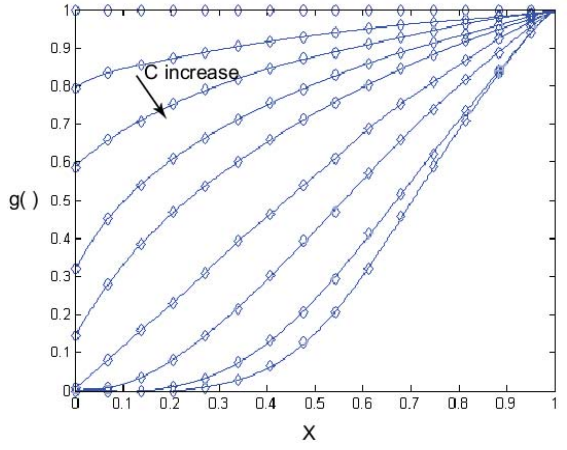

Figure 10. The measured static nonlinear function $g()$. The diamonds are the mearsured points. The curves are the polynomially fits from the mearsured points.

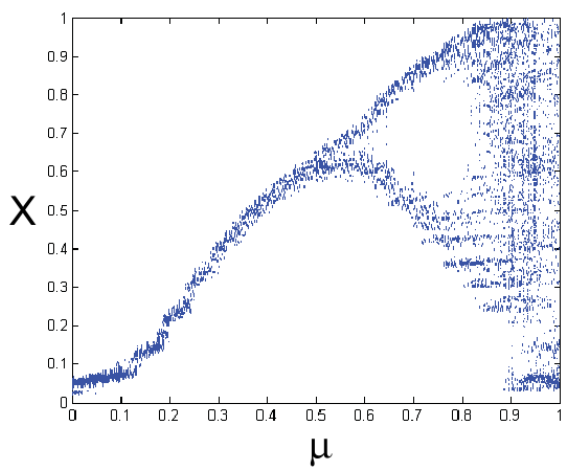

Figure 11. The measured bifurcation diagram from a logistic IRON

form the basis for the realization of a large-scale system-onchip for classification of complex spatio-temporal patterns.

\section{REFERENCES}

[1] E. Kandel, J. Schwartz and T. Jessell, Principles of Neural Science, 4thEdition, McGraw-Hill, 2000.

[2] N. Farhat, "Corticonics: the way to designing machines with brainlike intelligence", Proceedings of SPIE, Vol. 4109, pp. 103-109, 2000.

[3] Emilio Del Moral Hernandez, Geehyuk Lee and Nabil H. Farhat, "Analog Realization of Arbitrary One-Dimensional Maps", IEEE Transactions on Circuits and Systems I, vol. 50, pp. 1538-1547, Dec. 2003

[4] University of Pennsylvania patent disclosure, UOP-404US, Mar. 2005, Pending.

[5] J. Yuan, N. Farhat, J. Van der Spiegel, "A CMOS Monolithic Implementation of a Nonlinear Element for Arbitrary 1-D Map Generation”, ISCAS2006, pp. 2765-2768, Kos Island, Greece, May 2006.

[6] J. Yuan, N. Farhat, J. Van der Spiegel, "A CMOS Monolithic Implementation of a Nonlinear Interconnection Module for a Corticonic Network", ISCAS2006, pp. 2769-2772, Kos Island, Greece, May 2006. 